\title{
Du renforcement de l'assertion à la concession : variations
} d'emploi de certes

M. Jean-Michel Adam

Citer ce document / Cite this document :

Adam Jean-Michel. Du renforcement de l'assertion à la concession : variations d'emploi de certes . In: L'Information Grammaticale, N. 73, 1997. pp. 3-9.

doi : 10.3406/igram.1997.3555

http://www.persee.fr/doc/igram_0222-9838_1997_num_73_1_3555

Document généré le 16/10/2015 


\title{
DU RENFORCEMENT DE L'ASSERTION À LA CONCESSION : VARIATIONS D'EMPLOI DE CERTES
}

\author{
Jean-Michel ADAM
}

Le morphème CERTES n'a été que très brièvement décrit, dans des articles portant sur la dérivation (J.-C. Anscombre 1981 : 117-118), sur la polyphonie (O. Ducrot 1984 : 229230), sur la concession (M.-A. Morel 1996 : 16, 18, 78), sur les " marqueurs d'intention stratégique " (T. Nguyen 1984 : 45-78) ou encore sur les constructions périodiques complexes ou parenthésages ( $\mathrm{M}$. Charolles 1986 : 87-90). En m'appuyant sur une approche diachronique récente d'A. Rodríguez Somolinos (1995: 51-64) et sur le vaste corpus que j'ai réuni ces dernières années, je me propose d'affiner une description esquissée ailleurs (Adam 1990: 211-226).

\section{ESCRIPTIONS HABITUELLES DE CERTES}

Lies analyses de Ducrot, Nguyen et Morel mettent l'accent :U des enchainements CERTES $P$ MAIS $Q$ de ce type:

(1) Certes, il est beau de rêver l'éternité, maIS il suffit à l'honnête homme d'avoir passé en faisant son œuvre.

(Zola, Discours à la jeunesse, Écho de la Semaine du 28 mai 1893)

(2) La syntaxe didactique, telle que nous la concevons [...] est [...] beaucoup plus descriptive et analytique que normative ou doctrinale. CERTES, nous ne l'ignorons pas, en matière de syntaxe, comme dans la technique de tout art, il y a des règles, et dont quelques-unes s'imposent impérieusement à l'observation de chacun. MAIS, dans les choses de langue, ce qu'il y a surtout, ce sont des usages [...].

(G. Le Bidois, Préface de la Syntaxe du français moderne: 15)

Dans ces constructions, CERTES souligne le premier mouvement de la structure concessive. Polyphoniquement, une première proposition $[\mathrm{P}]$ est attribuée à un énonciateur dont le locuteur se distancie plus ou moins nettement. Ouvrant le second mouvement de la concession, MAIS a pour charge d'introduire une proposition $Q$ prise plus franchement en charge, elle, par un énonciateur avec lequel le locuteur s'associe. C'est aussi bien le cas de Zola, en (1), que de Le Bidois, en (2). Après avoir admis la vérité d'une première proposition, ils assertent avec plus de force la (les) proposition(s) introduite(s) par MAIS. Le premier mouvement, marqué par CERTES, est considéré par Ducrot comme l'acte de concession proprement dit, acte grâce auquel : «On peut se construire le personnage d'un homme à l'esprit ouvert, capable de prendre en considération le point de vue des autres » (1984:231).

Charolles et Anscombre, qui envisagent quant à eux le cas des emplois absolus de CERTES, reconnaissent l'existence de " deux usages principaux : un usage de renforcement, où il est très proche de certainement [...] et un usage concessif, où il est très fréquemment combiné avec mais " (Anscombre 1981: 117). Tels sont les deux sens retenus également par les dictionnaires contemporains:

$\checkmark 1$. (Indiquant ou renforçant une affirmation, positive ou négative). Certainement, assurément, bien sûr. Oui, CERTES! Non, CERTES! Ah, CERTES non! CERTES, je le vois bien.

REM. Au XVIIe s., CERTES vieillissait.

$\checkmark 2$. (Indiquant une idée de concession, d'opposition). CERTES, je n'irai pas jusqu'à prétendre que...

Souvent en relation avec mais, néanmoins. CERTES je voudrais le croire, mais je ne le peux.

REM. Sans être archaïque, CERTES est marqué, en français moderne, comme régional ou légèrement affecté; on emploie plutôt, selon les valeurs, certainement, sûrement, bien sûr. (Le Robert)

certes adv. 1. Exprime ou renforce une affirmation (soutenu): "Avez-vous lu ce roman? - Certes, et je l'aime beaucoup." [...]-2. Souligne une concession, une opposition : Je ne veux certes pas vous décourager, mais l'entreprise me semble bien difficile. On peut certes y aller en voiture, pourtant il serait dommage de ne pas marcher un peu (syn. ÉVIDEMMENT, ASSURÉMENT, BIEN SÛR, SANS DOUTE).

(Dictionnaire Larousse du français contemporain \& Pluridictionnaire)

II ressort de ces descriptions deux conclusions que je me propose de réexaminer : l'emploi isolé serait vieillissant (dès le XVIIe siècle), affecté, régional ou soutenu et ce connecteur serait, en français moderne, plutôt un concessif ouvrant une structure argumentative qui aurait besoin d'être fermée (parenthésage selon Charolles) par un autre connecteur explicite ou non : “ Même si le mais n'est pas explicité, il est attendu dès que l'on dit $P$, certes ou Certes, $p$ " (Anscombre 1981 : 118). En d'autres termes, une contrainte de complétude s'exercerait sur les suites de propositions comportant un CERTES et sa seule présence manifesterait l'existence de certaines réserves dans l'esprit du locuteur. C'est bien le cas dans cet éditorial d'Alain Peyrefitte, dans le Figaro Magazine du 5 mai 1984 (p. 159) :

(3) Le Président a CERTES fait preuve d'une louable détermination en se rendant sans délai à Beyrouth après l'attentat qui avait coûté la vie à plus de cinquante de nos militaires. L'opinion est sensible au panache. [...]

Le fait que le connecteur CERTES soit, en français contemporain, très souvent accompagné par un autre connecteur 
aboutit au fait que lorsqu'on litces lignes, on attend un renversement qui ne manque pas de venir aussitôt :

(3 suite) [...] Le Président a su faire diversion en jouant sur la fibre patriotique. II a satisfait dans le public un désir instinctif de vengeance en ordonnant des représailles. MAIS n'aurait-il pas dû se montrer ferme avant les attentats, plutôt qu'en être réduit, après, à frapper des innocents - le chauffeur de camion étant mort et les responsables à l'abri?

Mais en va-t-il de même, par exemple, dans cette citation des Mythologies (1957: 195) de Roland Barthes proposée dans le corpus informatique du Robert?

(4) Limage est, CERTES, plus impérative que l'écriture, elle impose la signification d'un coup, sans l'analyser, sans la disperser.

Si l'on isole cette phrase, l'argumentation semble être uniquement assertive et CERTES pourrait être remplacé par CERTAINEMENT OU ASSURÉMENT. Mais en se reportant au texte des Mythologies, on constate que le mouvement argumentatif de Barthes ne s'arrête pas là. L'assertion de (4) est modifiée dès la phrase suivante:

(4 suite) mals ceci n'est plus une différence constitutive. L'image devient une écriture, dès l'instant qu'elle est significative.

Si une certaine réserve, et donc une incomplétude, est bien sensible en (5), ce n'est pas le cas en (6), (7) ou (8). Le CERTES de (5) supporterait d'être remplacé par TOUTEFOIS, mais pas par CERTAINEMENT OU EN VÉRITÉ, qui conviendraient, en revanche, aux trois autres exemples:

(5) Et pour couronner le tout, Helena Bonham-Carter vient de quitter la maison de ses parents. Bon, d'accord, elle y faisait son lit elle-même, payait le loyer et avait le droit de recevoir. Certes, l'appartement où elle vient tout juste d'emménager est à dix minutes du domicile familial... le cordon n'est pas déchiré. Ou un autre va se créer.

(Eurostarmagazine, décembre 1996: 16)

(6) Le suicide est de trois natures: il y a d'abord le suicide qui n'est que le dernier accès d'une longue maladie et qui CERTES appartient à la pathologie ; puis le suicide par désespoir, enfin le suicide par raisonnement.

(Baizac, Illusions perdues, Pléiade, t. IV : 1013)

(7)

Que l'homme est né pour le bonheur,

CERTES toute la nature l'enseigne. [p. 176]

[...] Que l'homme est né pour le bonheur, CERTEs toute la nature l'enseigne. C'est l'effort vers la volupté qui fait germer la plante, emplit de miel la ruche, et le cœur humain de bonté. [p. 177]

(Gide, Les Nouvelles Nourritures, I, fragment qui reprend l'exergue de la p. 176 dans le paragraphe conclusif de la page suivante)

(8) II mourait d'épuisement, et CERTES, sans l'arrivée du reporter et de ses compagnons, c'en était fait de Cyrus Smith.

(Jules Verne, Lîle mysterieuse, t. I: 90)

En ne considérant pas ces derniers emplois autonomes de CERTES comme des exceptions, je me propose de repartir de l'histoire de la langue. Un éclairage diachronique nous aidera à reconsidérer la solution d'Anscombre qui, pour sauver le principe d'isomorphisme à la base de toute recherche de description unifiée, considère le CERTES du français moderne comme un marqueur de dérivation:
Certes dériverait un acte de concession d'un renforcement de l'assertion: on peut trouver bizarre qu'il en soit ainsi. Ce n'est rien d'autre que le simple fait que renforcer une assertion, c'est par là-même signaler que cette assertion avait besoin de l'être, et qu'elle pouvait donc être combattue. C'est de cette façon qu'une marque de renforcement devient une marque de concession. (Anscombre 1981: 118).

\section{DÉTOUR DIACHRONIQUE}

En français médiéval, CERTES n'est jamais employé totalement seul. II assure le renforcement de l'assertion réalisée ci-dessous par "oil » (je reprends des exemples d'A. Rodríguez Somolinos):

(9) Di jou voir, dame Mancevaire?

- Oil certes, dame Porrete. (Courtois d'Arras, 171)

Un tel « mode emphatique d'assertion que le locuteur prend entièrement à sa charge " (Rodríguez Somolinos 1995: 57) n'est pas du tout concessif. CERTES ne semble jamais avoir cette valeur en ancien français, même s'il est parfois - beaucoup plus rarement qu'aujourd'hui - employé en corrélation avec un connecteur concessif ou réfutatif:

(10) Et donc sui je ses anemis?

- Nenil, certes, MEs ses amis. (Chrétien de Troyes, Yvain 1460)

(11) Et si sachiez bien que ce n'a pas esté par ma chevalerie, mes par mon pechié; si m'avez fet dire ma honte. - Certes, biaus niés, fet li rois, voirement a ce esté mescheance droite, et je sei bien que ce vos est avenu par vostre pechié, MES TOUTEvoles me dites se vos cuidiez avoir ocis le roi Baudemagu. (La Mort le Roi Artu 3, 25)

Selon Amalia Rodriguez Somolinos : “ II s'agit toujours du même emploi de la modalité assertive : certes marque le point de vue du locuteur par rapport à la valeur de vérité de $p$, qui est de considérer $p$ vraie et de l'affirmer explicitement " (1995: 61). Commentant l'emploi de CERTES AINZ en français médiéval, elle souligne fort justement que le CERTES médiéval serait beaucoup mieux traduit par assurément, en vérité et CERTES AINZ par bien au contraire. Ainsi dans cet exemple:

(12) Ne cuidiez pas que ge vos mente, [Ne croyez pas que je vous mente] / certes, sire, ainz vos di voir [...]. [bien au contraire je vous dis la vérité]

(Jean Renart, Le roman de la Rose ou de Guillaume de Dole, 3337)

Cette valeur assertive, dominante en français médiéval, explique probablement le fait que, pour Littré, le sens de CERTES ne soit pas concessif:

certes /sèr-t'i adv. Certainement, en vérité, à coup sûr. En quoi certes personne ne le surpassa jamais, Vaugelas, dans Bouhours. Certes, messieurs, le barreau n'a vu que trop de ces malheureux, Patru, Plaidoyer, dans Bouhours, Nouv. rem. Mais certes c'en est trop d'aller jusqu'à la joie, Corn. Hor. I, 1. Certes l'exemple est rare et digne de mémoire, id. ib. IV, 2. [...] Elle lui respondit magnanimement certes, sans se troubler ny estonner, Amyot, Dion, 25.

Littré ne fait allusion à aucun emploi concessif. Le Larousse du xxe siècle (de 1929) est conçu sur un même modèle en faisant remonter la valeur assertive à son origine latine : 
CERTES [sèrt'] adv. (lat. certas). Certainement, assurément : Oui, CERTES. CERTES pour un amant la fleurette est mignonne. (Molière) // En poésie, on peut supprimer l's : CERTE on peut parler de la sorte. (V. Hugo)

Les auteurs du Dictionnaire historique de la langue française (Le Robert 1992 : 379) confirment l'étymologie latine, mais mentionnent les deux valeurs modernes en considérant toutefois la forme comme vieillie et affectée :

CERTES adv. est issu (1050) d'un latin populaire certas, accusatif féminin pluriel de certus ( $\rightarrow$ certain, certificat) ayant remplacé, avec une valeur adverbiale d'affirmation, l'adverbe classique certo "certainement», ablatif de certus. [...].

- Jusqu'au XVle siècle, le mot est usuel et possède plusieurs nuances, " sérieusement, fixement, attentivement "... II s'emploie dans des locutions comme à certes (1), pour certes, également à droites certes " dans le même sens", qui ont disparu. Au XVIe siècle, on y a vu à ce point une exclamation habituelle des Huguenots qu'on l'a employée par métonymie au sens de "Huguenot ". Considéré comme vieillissant, il s'est maintenu dans une valeur affirmative et concessive. Son emploi est aujourd'hui surtout affecté, à l'oral.

\section{EMPLOI AUTONOME DU CONNECTEUR POUR RENFORCER UNE ASSERTION}

En français moderne, CERTES peut, à la différence de la langue médiévale, constituer à lui seul une réplique. C'est l'emploi dit absolu bien représenté par (13), dans lequel l'exclamation souligne le caractère hyperthétique d'un évident renforcement de l'assertion. II peut également s'intégrer dans une réplique (14). Il peut aussi être employé de façon monologale, avec oui ou non/ne...pas (15):

(13) Le capitaine questionna le pilote:

- Connaissez-vous ces voiles?

- Certes ! répondit Gacquoil.

- Qu'est-ce?

- C'est l'escadre. (Gauthier, Capitaine Fracasse: 61)

(14) $-[. .$.$] II considérait sans déplaisir mes rapports avec Ethel..$ Si cela peut vous intéresser...

- Euh... Oui, CERTES. D'après ce que m'a dit Olivia, cette jeune personne pense beaucoup au mariage. [...]

(Floch \& Rivière, Le dossier Harding, Dargaud, p. 25, vignettes 2 \& 3)

(15) Quel aurait été, cependant, le sort de la 1re armée et des forces britanniques et françaises placées à sa gauche, si, vers sa droite, ne s'était ouverte, inopinément, la plaie béante de la Meuse? Je ne me sens CERTES pas la compétence nécessaire pour prophétiser là-dessus, après coup. (Marc Bloch, L'Étrange défaite., Folio-histoire, 1990: 70-71)

La valeur première de CERTES apparaît dans tous ces emplois. Dérivée du latin certas, cette forme pourrait être paraphrasée dans tous les cas de ce type par CERTAINEMENT, ENi VÉRITÉ, À COUP SÛR, BIEN SÛR, ÉVIDEMMENT Ou la périphrase IL EST VRAI QUE. Les emplois “ monorème " de CERTES permettent de comprendre son fonctionnement. Bally (1965b : 99) range CERTES dans les adverbes modaux (avec

1. À propos de cet emploi, on trouve, dans Littré, les exemples suivants: "Vous voyez bien que c'est à certes [pour de bon], quand de rechef s'est apparu l'ange vers moi ". "II ne pouvait croire qu'ils eussent parlé à certes [sérieusement] d'une si vaine matière". peut-être, naturellement, etc.) qui, pour la plupart, sont d'anciennes coordonnées adjointes ou incises : «ll s'agit partout de phrases monorèmes, plus ou moins exclamatives, qui interrompaient le courant de la phrase et ont fini par y être absorbées. Mais elles reprennent, à l'occasion, leur indépendance: Vous avez - oh! sûrement - entendu parler de la chose " (id. : 57). La définition de l'énoncé monorème comme une "phrase à un seul terme articulé " (id. : 53) et " exposant d'une pensée logiquement complète" (id. : 54) rend assez bien compte des emplois absolus du type de (13). Elle permet aussi de rendre compte des emplois dans des répliques un peu plus développées:

(16) Je ne l'ai jamais vu, ce qui ne l'empêche pas d'être mon maître.

- Et si tu le voyais, lui obéirais-tu ?

- Certes. Je serais donc un païen, si je ne lui obéissais pas! on doit obéissance à Dieu, et puis au roi qui est comme Dieu, et puis au seigneur qui est comme le roi.

(Hugo, Quatre-vingt-treize, p. 70)

L'incise monorème peut se fondre dans le reste de l'énoncé par la suppression des marques qui l'isolent : pause et intonation à l'oral, ponctuation forte à l'écrit (14) et (16). Cet effacement progressif est sensible d'abord dans la séparation par une simple virgule ou par un couple de virgule (17), puis dans la fusion typographique avec le reste de l'énoncé (18):

(17) Jurez de ne plus vous battre pour moi. Jurez-le si vous m'aimez comme vous le dites.

C'est un serment que je ne puis faire, dit le Baron; si quelque audacieux ose vous manquer de respect, je le châtierai, CERTES, comme je le dois, fût-il duc, fût-il prince.

(Gauthier, Capitaine Fracasse : 269)

(18) Après le potage la servante apporta une poule bouillie ; magnificence qui fit dilater les paupières des convives, de telle façon qu'elles semblaient prêtes à se fendre.

- On voit que vous aimez votre famille, Madame Coquenard, dit le procureur avec un sourire presque tragique ; voilà CERTES une galanterie que vous faites à votre cousin.

(ld. : 348)

Dans ces emplois dialogaux, le connecteur souligne l'accord du locuteur soit avec le propos de son interlocuteur, soit avec le sien propre, soit encore avec une opinion posée comme admise. CERTES marque la prise en charge, par le locuteur, d'une proposition (ou d'un ensemble de propositions) attribuable(s) à un énonciateur. ll en va de même dans les emplois monologaux. CERTES y occupe différentes places : on le trouve en début de phrase - soulignant éventuellement, comme en (19), un glissement de la narration au discours indirect libre -, en début de paragraphe (20) ou de strophe (21) sans qu'il prenne pour autant la valeur concessive que prédisait étrangement Anscombre (1981: 117) :

(19) Kheir ed Dîn avait rencontré plus d'une fois l'adversaire qu'il venait d'abattre. II n'avait que mépris pour ce Moulay Hassan, indigne héritier de la prestigieuse dynastie des Hafcides. Homme chétif et de piètre apparence, on aurait dit qu'il ployait sous le poids de la couronne et du manteau royal de ses ancêtres. CERTES il était voué à la défaite et à l'humiliation en face du terrible pirate, maître de la Méditerranée!

(Tournier, La goutte d'or) 
(20) Certes, c'est un sujet merveilleusement vain, divers et ondoyant, que l'homme. II est-malaisé d'y fonder jugement constant et uniforme.

(Montaigne, Essais, I, I)

(21) - Certes, je sortirai, quant à moi, satisfait D'un monde où l'action n'est pas la sœur du rêve ; Puissé-je user du glaive et périr par le glaive ! Saint Pierre a renié Jésus... il a bien fait. (Baudelaire, Les Fleurs du mal, " Révolte ")

\section{ENTRE LES PÓLES ASSERTIF ET CONCESSIF : UN EMPLOI RECTIFICATIF DE CERTES}

Dans certains emplois monologaux autonomes et souvent en incise, il semble que la valeur d'acquiescement presque emphatique et de soulignement de l'assertion par CERTES s'affaiblisse. Ainsi dans les incises (22), (23) et (24), on peut parler de rectification d'une assertion, d'un mouvement correctif :

(22) Frappée par le caractère exceptionnellement grave de son histoire récente, la Belgique attendait, cette fois, un discours royal hautement symbolique. En choisissant de descendre dans l'arène, CERTES à sa manière, Albert II a voulu se rapprocher de son peuple. Et lui exprimer le double sentiment qu'il lui porte : sa compassion et son admiration.

(Le Soir, lendemain du discours de Noël 1996 du Roi)

(23) La stylistique des chefs-d'œuvre n'est qu'une application CERTES privilégiée - d'un savoir qui cesse de se confiner dans une neutralité prudente. D'autres objets ne manqueront pas de s'offrir, si l'on croit détenir un pouvoir de lecture apte à dominer adéquatement les phénomènes de langage les plus divers.

(J. Starobinski, La Relation critique, Gallimard, 1970: 39)

(24) La Squadra azzura n'a pas fait le détail devant l'URSS, CERTES à court de compétition

- ITALIE-URSS 4-1 (3-1)

L'incise soulignée par le couple de tirets ou de virgules apparaît comme hétérogène au flux de la phrase dans laquelle elle se trouve insérée. En (22), le locuteur nuance l'expression " descendre dans l'arène ", jugée probablement trop forte pour décrire le style royal. La rectification l'emportant sur la concession, il semble très difficile de remplacer CERTES par un concessif (et même par les correctifs ENCORE QUE, BIEN QUE et QUOIQUE dont parle M.-A. Morel : 1995 : 10-15). EA (23), l'incise atténue l'effet de "ne... que " en revalorisant la stylistique littéraire. En (24), la proposition introduite par le connecteur apparaît comme la reconnaissance, par le locuteur, d'une objection possible. La conclusion qui pourrait être tirée des deux premières lignes et du score final de ce match (supériorité du football italien) est corrigée, atténuée (sens restrictif). La comparaison avec (24') permet de comprendre les effets de sens liés à l'emploi du connecteur:

(24') Devant une équipe d'URSS à court de compétition,

la Squadra azzura n'a pas fait de détail

- ITALIE-URSS 4-1 (3-1)

Un énoncé comme (24') relativiserait d'emblée l'ampleur de la victoire italienne. Avec CERTES, cette restriction n'est pas mise en avant, mais placée en fin d'énoncé. CERTES ne fait que modaliser le propos initial en incitant le lecteur à tenir compte quand même d'un nouvel élément: la forme très relative de l'équipe soviétique. Néanmoins, le locuteur adhère aux deux aspects de l'information : l'évidence du bon résultat et l'objection restrictive. II prend acte du score final en le nuançant.

II semble en aller de même en (5) et dans les trois exemples suivants où CERTES, en tête de phrase ou de paragraphe, ouvre un développement rectificatif au sein d'un raisonnement ou d'une description d'un état de fait :

(25) Cette conception du style inspirera toute une série de définitions du style qui représentent la même tradition, selon laquelle, suivant le mot de Buffon, "le style, c'est l'homme même". CERTES, le contexte dans lequel figure cette phrase en limite quelque peu la portée.

(F. Deloffre, Stylistique et poétique françaises, C.D.U. SEDES, 1970)

(26) Pour ses créateurs, il sera un " surdoué des mers ", plus gros que le "Redoutable ", mais n'emportera que le même nombre de missiles : seize. Certes, ces missiles seront améliorés par rapport aux fusées actuelles. Ils seront " durcis ", sur le plan électronique, pour résister aux contre-mesures adverses. Et le nombre de têtes nucléaires dans chaque fusée pourra atteindre huit, permettant d'un seul coup une dispersion sur huit objectifs différents.

(La Nouvelle république du centre-ouest, 16.3.90, article consacré à la construction du sous-marin nucleaire "Triomphant ")

(27) [...] Le franc s'est inscrit à soñ plus haut cours vis-à-vis de la devise allemande depuis près de deux ans.

Certes, le mark n'est pas en très bonne posture actuellement, à la veille de la fusion monétaire entre la RFA et la RDA, qui fait craindre une surchauffe et un regain d'inflation. Par ailleurs, les capitaux étrangers, y compris japonais, affluent à Paris, attirés par les taux d'intérêt français [...].

(Le Monde, 30.3.90)

Dans tous ces emplois de CERTES, il semble possible d'affirmer que malgré la proposition CERTES $P$, le cotexte antérieur n'en reste pas moins entièrement vrai. Les exemples (25) et (26) admettent plus facilement que les précédents un remplacement de CERTES par BIEN QUE, TOUTEFOIS Ou CEPENDANT. Mais à la différence de l'emploi concessif qui tire CERTES vers la droite, vers l'aval cotextuel - comme nous allons le voir -, l'emploi rectificatif porte sur une proposition située textuellement à gauche, en amont du connecteur. En (26), comme en (23), la proposition introduite par CERTES corrige un ne... que précédent en revenant sur ce dont il vient d'être question (missiles ou stylistique littéraire) ; en (25), un retour est opéré sur la phrase de Buffon (ce que confirme l'anaphore "cette phrase "); en (27), c'est sur la monnaie allemande (relexicalisée : "le mark») que revient l'énoncé.

\section{UN EMPLOI FRANCHEMENT CONCESSIF}

Très proches de (22) et (23), les incises suivantes paraissent plus franchement concessives:

(28) De ces définitions, CERTES un peu désuètes, nous retiendrons un certain nombre de points.

(P. Guiraud, Essais de stylistique, Klincksieck, 1969) 
(29) Le plus curieux, c'est que la métamorphose ne lui déplaisait pas. A travers le masque du vieillard majestueux qui s'effritait, il voyait reparaître - plus accusés, plus marqués CERTES - les traits du jeune homme imberbe qu'il avait été.

(M. Tournier, Les Rois mages, Gallimard, 1980 : 112-113)

Une reformulation explicitement concessives donnerait :

(28') BIEN QUE ces définitions puissent paraître un peu désuètes, nous retiendrons un certain nombre de points.

(29') [...] II voyait reparaître - (TOUTEFoIS) plus accusés, plus marqués (CEPENDANT) - les traits du jeune homme imberbe qu'il avait été.

Bien qu'employé toujours de façon autonome, CERTES reprend, en (30) le « il est certain que » du paragraphe précédent et il entre en contradiction forte avec la fin du paragraphe :

(30) Certains auteurs ont tendance à restreindre le rôle des inducteurs à celui de stimulants banals et à exalter les propriétés des tissus compétents comme seuls responsables de la différenciation. II est certain que le tissu compétent est l'effecteur de la différenciation [...].

Le tissu compétent contient CERTES en puissance presque tout ce qu'il faut pour réaliser l'une ou l'autre différenciation; il lui manque cet apport encore mal défini que lui fournit l'inducteur. (E. Wolff, in Sciences, $n^{\circ} 1: 12-13$ )

On pourrait fort bien écrire: " [MAIS] il lui manque [CEPENDANT/TOUTEFOIS] cet apport... " afin de rendre le mouvement concessif plus explicite.

Un fonctionnement proche se retrouve dans cette conclusion d'un article de Libération. La proposition introduite par CERTES y redouble celle qu'introduit un très semblable " on conviendra volontiers ". Mais ici le renversement concessif est explicitement introduit :

(31) On conviendra volontiers que bien des maux dénoncés ici ne sont pas nouveaux [...], ou qu'ils ne sont pas propres au monde de l'architecture [...]. Et, CERTES, le libre marché et la démocratie représentative sont "les moins mauvais des systèmes " et la ville est aussi faite du chaos... Encore faudrait-il savoir si ce chaos est celui c'un libéralisme niveleur et ravageur ou s'il est libertaire et libérateur d'énergies positives. (26.10.1995, page 6)

Dans les structures ironiques, l'absence de tout connecteur concessif est normale : l'ironie est une procédure trop implicite et indirecte pour tolérer un marquage fort :

(32)Tout allait pour le mieux. Les écrivains écrivaient, les maisons d'édition publiaient, les critiques faisaient semblant de critiquer, les jurys couronnaient, les intrigues fleurissaient, les potins potinaient, les foires foiraient, les télévisions tournaient. CERTES, le niveau baissait, et le délit de littérature, le vrai délit d'initié, était de plus en plus rare. On commémorait, on s'arrachait les vieillards survivants, on les photographiait [...]. (Le Monde, 3.3.89, début d'un article de Philippe Sollers)

(33) Ne vous fiez pas aux apparences : j’ai des goûts très simples. [première page avec photographie d'une jeune femme extrêmement élégante]

CERTES, CERTES.

ldeuxième page avec photographie en gros plan d'une bouteille de champagne de la marque PIPER-HEIDSIECK qui vient confirmer les goût de luxe de l'élégante]
Ce dernier exemple publicitaire joue sur le renversement du verbal par liconique (condition élémentaire de la lecture ironique d'un énoncé), renversement que le redoublement dubitatif de CERTES se contente d'appuyer.

La question qui se pose à présent est clairement la suivante : est-ce le connecteur CERTES lui-même qui possède une valeur d'emploi concessive ou cette valeur est-elle engendrée par le cotexte et soulignée généralement par un connecteur concessif? CERTES n'aurait alors, dans tous les cas, pour fonction que de signaler une adhésion plus ou moins forte du locuteur à la vérité de la (des) proposition(s) qu'il introduit.

En français moderne, le fonctionnement concessif le plus fréquent de CERTES est un emploi lié, dans des parenthésages qui adoptent des corrélations variées : avec MAIS (1) à (4)-, NÉANMOINS (et ses variantes AU MOINS, DU MOINS, PAS MOINS), aveC TOUTEFOIS, POURTANT, CEPENDANT, EN REVANCHE.

(34)La Chrummiadenflue, située dans l'est du massif du Gantrisch, ne compte CERTES pas parmi les classiques de l'alpinisme à ski en Suisse. Son nom - qui signifie à peu près " Crête du Fil Courbe " - ne promet rien de grandiose. NEANMOINS, ce sommet d'un peu plus de 2000 mètres jouit d'une grande popularité dans les environs de Berne. Brièveté du déplacement motorisé ; montée limitée à $1 \mathrm{~h} 1 / 2$ à 2 heures, démarrant sur la route du Gantrisch à environ $1600 \mathrm{~m}$ d'altitude ; pente terminale attrayante et sommet aérien, rocheux; course facile réalisable dans presque toutes les conditions [...] (revue Les Alpes, 1er trimestre 1993)

(35) Certes, les Parmesans furent les plus dangereux sur l'ensemble de la rencontre et le PSG doit une fière chandelle à son gardien, auteur de quelques interventions assez rares mais décisives. IL N'EN RESTE pas moins QUE les Parisiens ont largement fait jeu égal et qu'ils furent le plus souvent en possession du ballon dans le dernier quart d'heure. (Le Matin, 8.3.1996).

(36) Certes, je n'aborde pas, de gaieté de cœur, cette partie de ma tâche. Français, je vais être contraint, parlant de mon pays, de ne pas en parler qu'en bien ; il est dur de devoir découvrir les faiblesses d'une mère douloureuse. Historien, je sais mieux que quiconque les difficultés d'une analyse qui, pour ne pas demeurer trop imparfaite, devrait remonter jusqu'aux ramifications causales les plus lointaines, les plus complexes et, dans l'état actuel des sciences humaines, les plus cachées. Qu'importent ici, CEPENDANT, de petits scrupules personnels.

(M. Bloch, L'Étrange défaite, Folio-histoire, 1990: 159-160)

(37) Est-ce là s'éloigner de l'œuvre? Oui CERTES, puisque je cesse d'être un lecteur docile, puisque mon trajet n'est plus régi par celui même de l'œuvre : je m'en détache et je m'en écarte pour suivre mon parcours propre. TOUTEFOIS ce parcours reste toujours relatif à l'œuvre avec laquelle tout à l'heure je coîncidais ; et l'éloignement que j'ai conquis m'apparaît comme la condition nécessaire pour qu'il n'y ait plus seulement acquiescement à l'œuvre littéraire, mais rencontre avec celle-ci.

(J. Starobinski, La Relation critique, 1970 : 11) 
( 38) Certes, les élus du Château font leur mea culpa. Bien sûr, ils prennent quelques mesures qui s'imposent. Effectivement, ils ne sous-estiment plus le désastre. EN REVANCHE, sont-ils "politiquement corrects "avec eux-mêmes et les électeurs? Le courage, le vrai courage politique eût été de démissionner collectivement et de se représenter devant les urnes.

(Le Matin, 5.3.1996)

Ce dernier exemple présente l'avantage de proposer en série trois assertions introduites par des synonymes de CERTES, avant que ne s'opère le renversement. Très souvent, la segmentation typographique souligne la séparation des deux mouvements de la concession par un alinéa. Ainsi dans ce passage de L'Étrange défaite de Marc Bloch :

(39) J'entends, chaque jour, prêcher par la radio, le " retour à la terre ». [...] CERTES, je n'ignore pas que sous ces beaux sermons se dissimulent - en vérité assez mal - des intérêts bien étrangers au bonheur des Français. Tout un parti, qui tient aujourd'hui ou croit tenir les leviers de commande, n'a jamais cessé de regretter l'antique docilité qu'il suppose innée aux peuples modestement paysans. On pourrait bien s'y tromper, d'ailleurs. Ce n'est pas d'hier que nos croquants ont, comme disaient les vieux textes, "la nuque dure ". Surtout l'Allemagne, qui a triomphé par la machine, veut s'en réserver le monopole. C'est sous l'aspect de collectivites purement agricoles contraintes, par suite, d'échanger, à des prix imposés, leurs blès ou leurs laitages contre les produits de sa grande industrie, qu'elle conçoit les nations, dont elle rêve de grouper autour d'elle, comme une valetaille, l'humble compagnonnage. À travers le micro, la voix qui parle notre langue vient de là-bas.

Ces bucoliques avis, POURTANT, ne sont pas exclusivement choses d'aujourd'hui. Toute une littérature de renoncement, bien avant la guerre, nous les avait rendus déjà familiers.

(1990: 180)

II n'est pas rare, comme en (39), que l'on soit obligé de pousser l'investigation assez loin pour trouver le renversement de la première assertion par une nouvelle qui la transforme rétroactivement en concession. Ainsi dans "Lazzara ", long poème des Orientales, Hugo repousse de trente vers un renversement qui n'est souligné que par une négation. Ce renversement est toutefois attendu en raison du marquage des propositions par un irréel du passé (subjonctif plus-queparfait qui souligne clairement la non-adhésion du locuteur à la vérité des propositions) :

(40) Certes, le vieux Omer, pacha de Négrepont, [vers 25]

Pour elle eût tout donné, vaisseaux à triple pont,

Foudroyantes artilleries,

Harnois de ses chevaux, toison de ses brebis,

Et son rouge turban de soie, et ses habits

Tout ruisselants de pierreries;

Et ses lourds pistolets, ses tromblons évasés, [...]. [v. 31]

Il eût donné sa housse et son large étrier ; [...] [v. 37]

II eût donné les Francs, les Juifs et leur rabbin; [...] [v. 43]

Tout ! jusqu'au cheval blanc, qu'il élève au sérail, [...] [v. 49]

Ce n'est point un pacha, c'est un klephte à l'œil noir [v. 55]

Qui l'a prise, et qui n'a rien donné pour l'avoir.

Car la pauvreté l'accompagne;

Un klephte a pour tous biens l'air du ciel, l'eau des puits,

Un bon fusil bronzé par la fumée, et puis

La liberté sur la montagne.
Pour comprendre le fonctionnement du mouvement concessif, considérons rapidement (40) et cette légende d'une photo de presse :

(41) Dominique Perret, skieur extrême: " Nous ne sommes pas des kamikazes suicidaires : CERTES; nous.prenons des risques, mals ils sont calculés ".

(Le Nouveau quotidien, 24.5.1994)

En (41) comme dans la plupart des cas - et malgré les exemples (5), (25) ou (26) - on peut difficilement prétendre que CERTES marque à lui seul la concession. C'est la combinaison de CERTES et d'un autre connecteur qui rend possible le mouvement complexe suivant: CERTES souligne que la proposition P ("nous prenons des risques") est un argument pour une conclusion sous-jacente à la négation de la proposition précédente : on pourrait croire que "nous [...] sommes [...] des kamikazes " (C). L'interprétant est invité à déduire cette conclusion de la proposition négative. À lui tout seul, CERTES ne peut que souligner le mouvement d'adhésion à l'enchaînement [proposition CERTES $P>$ donc $>$ conclusion C]. La combinaison CERTES-MAIS place CERTES sous l'influence d'un mouvement argumentatif que l'on peut ainsi décrire.

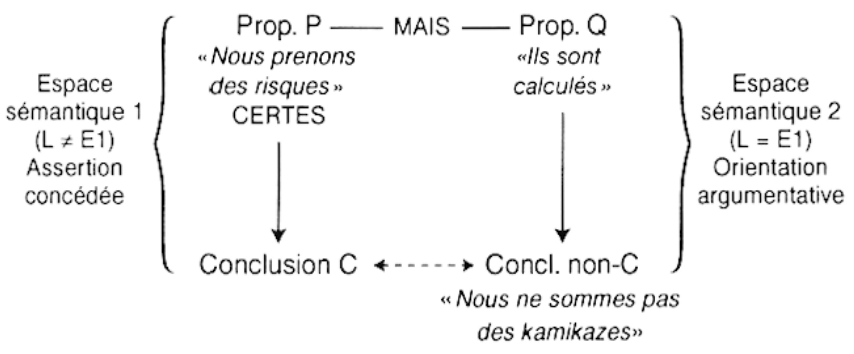

De la même manière, dans le poème de Hugo, après les quatre premières strophes, qui ont décrit l'extrême beauté de Lazzara, on est tenté de conclure des cinq strophes contrôlées par CERTES : seule une extrême richesse permettrait de conquérir (acheter?) une telle beauté. Mais la dernière strophe agit comme une chute en renversant totalement cette attente et en entraînant une conclusion du type: la liberté l'emporte sur (vaut mieux que) toutes les richesses.

CERTES ne porte que sur le constituant-argument le plus faible, la cause présentée comme non déterminante. En soulignant un premier mouvement d'adhésion aux normes qui régissent l'espace sémantique d'un énonciateur $E 1$, l'assertion marquée par CERTES ne se transforme en concession que parce que cet espace sémantique est précisément celui que le locuteur (L), après avoir admis son existence et reconnu sa pertinence, ne va pas prendre en charge. $\mathrm{Ce}$ sont MAIS / POURTANT / EN REVANCHE / etc. qui assurent le mouvement argumentatif général en plaçant CERTES au service du seul premier mouvement - concessif - et en orientant, comme ci-dessus, le discours dans le sens de la conclusion NON-C.

Le recours à CERTES permet de ne pas disqualifier d'entrée un contexte normatif qu'il s'agit pourtant de contester. La description polyphonique de la prise en charge des propositions permet de rendre compte de la stratégie concessive. 
Dans un premier temps, un acte d'affirmation pose la vérité d'une assertion et l'on peut dire que la fonction de CERTES est de soutenir cette première assertion. Si la suite de l'énoncé ne renverse pas ce premier mouvement, l'on a affaire à un emploi (sur)assertif du connecteur. Si des propositions, introduites ou non par un connecteur concessif ou plus largement argumentatif, viennent renverser ce premier mouvement, l'interprétant est amené à recalculer le degré de prise en charge de CERTES P / P, CERTES par le locuteur.

L'approche polyphonique permet de rendre compte d'un enchaînement complexe de type CERTES PAS > AU CONTRAIRE :

(42) Le rappel de Rastignac tout au début du récit N'est CERTES PAS un détail négligeable, il faut AU CONTRAIRE le tenir pour essentiel en ce qu'il indique d'emblée le fonds d'idées et de souvenirs littéraires sur lequel Flaubert détache son roman. (Marthe Robert, En haine du roman, Poche-Balland 1982: 105)

Tandis que CERTES PAS appuie la proposition NON-P, AU CONTRAIRE ne peut s'opposer qu'à une proposition $P$ implicite (c'est un détail négligeable) attribuable à un énonciateur $E 1$ dont le locuteur se dissocie nettement $(L \neq E 1)$. La polyphonie garantit le caractère non absurde d'un tel enchaînement: c'est avec un énonciateur E2, prenant en charge NON-P que le locuteur marque son accord. La proposition introduite par AU CONTRAIRE ne vient pas contredire, comme c'est le cas dans le mouvement concessif, la proposition introduite par CERTES NE... PAS, elle vient seulement la renforcer : ce détail n'est pas négligeable, il est MÊME essentiel.

\section{POUR UNE DESCRIPTION GRADUELLE}

Après Charles Bally, il est intéressant de souligner la valeur assertive d'un CERTES qui rentre dans le paradigme des diffé rentes façons de dire OUI: "Certes! Ma foi oui! Mais oui! Pourquoi pas ? Parfaitement! À quile dites-vous ? Je l'avoue! J'en conviens! D'accord! C'est dit! Soit ! Amen! etc. " (Bally 1965 : 20). Série à laquelle nos contemporains semblent décidés à ajouter aujourd'hui un envahissant "Tout à fait ». Dans cet esprit, CERTES ne permet que d'attribuer une proposition à un énonciateur ( $E 1)$. Un énoncé CERTES $P$ (ou $P$, CERTES) signale que le locuteur adhère à une logique menant d'une proposition $\mathrm{P}$ à certaines conclusions qu'il attribue à un énonciateur - à son interlocuteur ou à lui-même ou à une opinion posée comme admise - $(L=E 1)$. Un affaiblissement de cet accord peut faire glisser CERTES de cette valeur première d'accord plein et entier en direction d'un emploi rectificatif, un affaiblissement supplémentaire mène doucement à la concession. Le fait qu'avec CERTES une proposition $P$ puisse être assertée indirectement par le locuteur (son assertion mentionnant celle d'un énonciateur $E 1$ ) rend très facile l'opération de distanciation propre à l'acte de concession. La valeur concessive ne semble toutefois pas pouvoir être attribuée en propre au connecteur CERTES : elle résulte d'un effet soutenu la plupart du temps par un parenthésage résultant de l'action sur CERTES d'un autre connecteur. Tout dépend, en fait, de degré de vérité accordé par le locuteur au propos qu'il attribue polyphoniquement à E1 : moins l'adhésion du locuteur est forte, plus l'interprétation concessive devient plausible. C'est une affaire de degrés, sur une échelle d'adhésion qui permet de classer tous nos exemples (tableau ci-dessus).

La variation diachronique semble bien se doubler d'une variation synchronique dans les emplois possibles de CERTES.

\section{Jean-Michel ADAM Université de Lausanne, Centre de recherches en linguistique textuelle et analyse des discours}

\section{RÉFÉRENCES BIBLIOGRAPHIQUES}

Adam, J.-M., 1990. Eléments de linguistique textuelle, Mardaga, coll. Philosophie et langage, Liège.

Anscombre, J.-C., 1981. "Marqueurs et hypermarqueurs de dérivation illocutoire: notions et problèmes ", Cahiers de linguistique française $n^{\circ} 3$, Université de Genève.

Bally, Ch., 1965 (1925). Le langage et la vie, Droz, Genève. - 1965b (1944). Linguistique générale et linguistique française, A. Francke, Berne.

Charolles, M., 1986. "La gestion des orientations argumentatives dans une activité rédactionnelle ", Pratiques $n^{\circ} 49$, Metz.

Ducrot, O., 1984. Le dire et le dit, Minuit, Paris.

Morel, M.-A., 1996. La concession en français, Ophrys, GapParis.

Nguyen, T., 1984. "Sens et intentions ", Semantikos vol. 8, $n^{\circ} 1$, Paris.

Rodriguez Somolinos, A., 1995. "Certes, voire : l'évolution sémantique de deux marqueurs assertifs de l'ancien français ", LINX $n^{\circ} 32$, Université de Paris X-Nanterre.

\begin{tabular}{|c|c|c|c|c|c|c|}
\hline PÔLES & $\begin{array}{l}\text { ASSERTIF } \\
\text { Adhésion } \\
\text { maximale }\end{array}$ & + & $\begin{array}{c}\text { Gradation de la } \\
\text { distance énonciative }\end{array}$ & & $\rightarrow$ & $\begin{array}{l}\text { CONCESSIF } \\
\text { Distanciation } \\
\text { maximale }\end{array}$ \\
\hline EXEMPLES & $\begin{array}{c}6 \text { à } 8 \\
13 \text { à } 21\end{array}$ & $\begin{array}{c}24,27 \\
42\end{array}$ & $\begin{array}{l}22 \\
23\end{array}$ & $\begin{array}{c}5 \\
25,26\end{array}$ & \multicolumn{2}{|c|}{$\begin{array}{c}1 \text { à } 4 \\
28 \text { à } 41\end{array}$} \\
\hline
\end{tabular}

